\title{
A Study on Image Segmentation Method for Image Processing
}

\author{
S. PRABU ${ }^{\mathrm{a}}$ and J.M. GNANASEKAR ${ }^{\mathrm{b}}$ \\ ${ }^{a}$ Associate Professor Department of Computer Science \& Engineering, VI Institute of \\ Technology Sirunkundram-603108, Chengleput Distirct, TamilNadu, India \\ ${ }^{b}$ Professor, Department of Computer Science \& Engineering, SriVenkateswara \\ College of Engineering, Sriperumbadur, TamilNadu, India
}

\begin{abstract}
Image processing techniques are essential part of the current computer technologies and that it plays vital role in various applications like medical field, object detection, video surveillance system, computer vision etc. The important process of Image processing is Image Segmentation. Image Segmentation is the process of splitting the images into various tiny parts called segments. Image processing makes to simplify the image representation in order to analyze the images. So many algorithms are developed for segmenting images, based on the certain feature of the pixel. In this paper different algorithms of segmentation can be reviewed, analyzed and finally list out the comparison for all the algorithms. This comparison study is useful for increasing accuracy and performance of segmentation methods in various image processing domains.
\end{abstract}

Keywords. Image Segmentation, Digital Image Processing, K-Means Clustering, Edge detection, histogram.

\section{Introduction}

Image is binary representation of visual information by means of pixels. It contains lot of information to perform some useful operations on different area of applications like medical field, object detection, video surveillance system, computer vision, pattern recognition, remote sensing etc. With the help of computer algorithms, we can manipulate the pixels either enhance the quality of image or extract the useful information from it. Digital image processing has different stages, in which segmentation is the essential and challenging part in the operation of image processing. Image segmentation should be segregating the images into meaningful parts that are having similar features and behaviors. The purpose of segmentation is, to make the image representation easily, classified meaningfully, and analyzed properly. Using the image segmentation, we can localize the objects and identifying its boundaries in an image. Then assign the labels to each pixel in the entire image. Some pixels having the same labels means they have common characteristics. This paper analyzes the different methods of segmentation and their algorithms. First, we analyze the segmentation carried out by means of pixel properties [1] to analyze the local and global properties, and to tell how speed up the segmentation process by modifying the existing ACM model along with local and global properties. Expectation of maximization algorithm [2] needs more iteration to segment the image. Modification can be based on the

\footnotetext{
${ }^{1}$ S. PRABU, Associate Professor Department of Computer Science \& Engineering, VI Institute of Technology Sirunkundram, India

Email: sprabumkm@gmail.com
} 
likelihood properties we can achieve the minimum number of calculations. EM based localization and 3D U-Net and EM based attention mechanism will provide the optimized segmentation results. Color images can be segmented by RGB Histogram [3] along with firefly algorithm. Combination of SCT-I and SCT-V algorithm and SAMFO-TH [17] algorithm solves the multilevel threshold problem of RGB color image. Color hymnographies [6] can be used to identify the color object detection. Image restoration algorithm [4] can segment the image based on the sample blocks. This method utilizes the advantages of both Criminisi algorithm \& Watershed image segmentation algorithm. So many factors are affecting while extracting text information from the image [5]. Noise is more, when the image can be captured from live video camera. The Encoder - Decoder framework resolves the issues in segmentation for retrieving text information. To speed up the text retrieval process, Instance Segmentation Network technique can be used. This paper analyze the different methods and compare it to give the clear idea about image segmentation process.

\section{Related Work}

\subsection{Segmentation by means of properties of pixel}

Segmentation operation can be also performed by means of global and local properties of pixels [1]. It states that, the global properties are calculated by mean values of various pixels and successive edges of objects, and the local properties are characterized by interaction of successive pixels and the boundary of the image. Image entropy can be done by improved weighting function that can be adapt the weight between local term and global term, so the segmentation speed can be improved significantly.

\subsubsection{Thresholding Method}

Based on the pixel's intensity, this method partitions the image, into two parts, they are intensity of pixel value lower than threshold and intensity of pixel value greater than threshold. Multi stage thresholding approach is necessary for color image segmentation in many applications [10]. To segment the color components Red, Green and Blue, more than two optimal thresholds can be needed. In future a greater number of thresholds is necessary. But a greater number of thresholds can decrease the performance of segmentation process. The main drawback of multi-level thresholding leads long execution time [11]. During the processing of low-quality images, cause the wrong prediction of threshold values. The improved salp swarm algorithm can minimize the number of thresholds to ensure effective segmentation process. Modified grasshopper optimization algorithm [16], metaheuristic algorithm, firefly algorithm, novel population-based bee foraging algorithm [18] are also used for optimized threshold values.

\subsubsection{Edge detection}

An edge is a set of connected pixels that creates the boundary between the two successive regions. The edge detection is basically a process of segmenting the image into discontinuity of regions. The perfectness of the image-by-image processing and computer vision can be depending on identifying meaningful edges. Out of all the edge detection techniques, 'Canny Edge Detector' produces good results than other edge detection methods. The basic limitations of edge detection are edge connectivity and edge thickness [7]. Multiple values of Threshold approaches need to solve the above two issues. To find the optimized thresholding values Genetic algorithm can be 
applied which optimize the coefficient of filter [8]. This kind of optimization improves the quality of segmenting edges in MR scan images.

\subsubsection{Clustering methods}

Clustering is the process of image information can be replaced by clusters. Cluster is the collection of similar attribute data points. Pixels, which have common attributes like same color, same texture or any related attribute. Main issues in Clustering are identify the correct inter cluster distance and identify the total number of Clusters. Robust self-sparse fuzzy clustering algorithm improves the clustering result by reducing the noisy features. The main drawback of $\mathrm{K}$ means clustering method is image segments are disconnected and disseminated with wide distance. To find the optimal number of clusters is very hard without knowing the initial parameters. The unsupervised K-means clustering technique is needed to achieve this [9]. To solve the initialization problem, UK-means algorithm uses number of points to determine the initial number of clusters. To avoid overlapping, kernel K-means clustering can be proposed [13]. In which kernel functions are transformed into feature space, to estimate the correct number of clusters. The combination of depth and semantic information of images [20] improve the accurate identification of initial center value.

\subsection{Optimized Segmentation}

Segmentation operation can be improved effectively by applying small changes in the existing segmentation algorithm called Expectation of maximization; it has a greater number of iterations and takes more computing power [2]. Compression based segmentation methods express the approximation of actual pixel values with some of the sample pixels, so that the size of the image can be tremendously reduced. To improve the efficiency of compression, the original image can be pre quantized with higher bits [15]. But sometimes the predictions may not work properly when using high bit rates.

\subsection{Color Image Segmentation}

Segmentation operation can be achieved in a color images by RGB Histogram [3]. By applying firefly algorithm, the optimal multi-level image segmentation can be achieved. The two new algorithms called SCT-I and SCT-V for image and video input data. For each frame SCT-V algorithm locate the target of interest (TOI) for object tracking and SCT-I algorithm maintain the original color in the target of interest. SAMFO-TH algorithm [17] based on moth flame optimization. Histogram is developed by dividing the range of the data into same sized classes. Then for each class, those data set points which present into the class are calculated. The key parameter of this method is the selection of the threshold value, which can be computed manually or automatically by some algorithms. The basic concept of this method is, to identify the mean or median value, so that pixels of the object are brighter than the background. By applying color hymnographies to increase the color fidelity and improve the color object detection [6].

\subsection{Segmentation based on the sample blocks}

Segmentation operation can be achieved by improved image restoration algorithm based on the sample blocks [4]. It resolves the image repairing problems occurred when the image restoration process. Criminisi algorithm and watershed image algorithm is applied to the large amount of image set. Then identify the matching pixel 
blocks in image segmentation. So that excessive extension of texture blocks for the process of restoration of images can be avoided.

\subsubsection{Region-based methods}

This kind of segmentation groups the pixels that have similar properties. It segregates the pixel, those having the similar characteristics and dissimilar characteristics. It compares the properties of neighboring pixels and produces the result. The main objective is to differentiate the homogeneity of the image. That can be achieved by improved image restoration algorithm based on the sample blocks [4]. A new method using region of interest for segmenting images, having less computation complexity, to preprocess the training sets so that to reduce the redundant information. For color region segmentation, hue division based selective color transfer algorithm can be used. In which HSV color model transferred into luminance and saturation integrity.

\subsection{Text-Based Segmentation}

Segmentation operation can be achieved only in a text-based images, to retrieve the necessary text information from the whole image [5]. The information may be single or multiple line, words, or even one or more characters. This paper proposes different methodologies at the various segmentation stages. It first justifies the segmentation process in the text context based on the information retrieval. This paper also discusses different factors affecting the process of segmentation. The Captured images from camera have more background noise [12]. The main issues are symbols having several separate primitives with complex background and distortions from camera. To extract the text information from natural scenes are also complex process. The encoder - decoder framework [17] is proposed. It is used by the combination of attention mechanism and connection time classification. To reduce the more processing time the new technique called Instance Segmentation Network (ISNet) to detect the text content by generating prototype masks simultaneously.

\subsection{Motion \& Interactive Segmentation}

Motion Segmentation means pixels are grouping together in a particular movement of the object. The main objective of this technique is to segment the images for object that are in moving condition. To make the image analysis, and understanding the image properties, to analyze the motion sequence is important. First Identify the background and foreground objects, and then make analysis these objects independently, this model is commonly called 'variation Model' which takes two successive video frames, evaluate the movement ranges between each frame. The main aim of this method is to identify the object boundaries with minimal user interventions.

\section{Survey Discussion}

Various segmentation algorithms are discussed and each method has its own advantages and disadvantages within a particular context. Some methods need the modification and improvement. The following table 1 compares the various factors of different segmentation methods. 
Table 1: Comparison of different segmentation techniques

\begin{tabular}{|c|c|c|c|c|}
\hline Method & $\begin{array}{l}\text { Descripti } \\
\text { on }\end{array}$ & Characteristics & Advantages & Issues \\
\hline $\begin{array}{l}\text { Threshold } \\
\text { ing } \\
\text { Method }\end{array}$ & $\begin{array}{l}\text { Segmentation } \\
\text { done by value } \\
\text { of } \quad \text { pixel } \\
\text { Intensity }\end{array}$ & $\begin{array}{l}\text { Types: } \\
\text { Local \& Global } \\
\text { Thresholding }\end{array}$ & $\begin{array}{l}\text { Simple \& Effective } \\
\text { Processing. }\end{array}$ & $\begin{array}{l}\text { Single Threshold value } \\
\text { not give accurate } \\
\text { result. More Threshold } \\
\text { values leads more } \\
\text { processing time }\end{array}$ \\
\hline $\begin{array}{l}\text { Edge } \\
\text { detection }\end{array}$ & $\begin{array}{l}\text { Segmentation } \\
\text { based on } \\
\text { discontinuity of } \\
\text { regions }\end{array}$ & $\begin{array}{l}\text { Steps } \\
\text { Filtering } \\
\text { Enhancement } \\
\text { Detection } \\
\end{array}$ & $\begin{array}{l}\text { Using probability for } \\
\text { finding error rate, } \\
\text { Improving signal to } \\
\text { noise ratio. }\end{array}$ & $\begin{array}{l}\text { Highly sensitive to } \\
\text { noise. } \\
\text { More possible for } \\
\text { inaccuracy. }\end{array}$ \\
\hline $\begin{array}{l}\text { Clustering } \\
\text { methods }\end{array}$ & $\begin{array}{l}\text { Segmentation } \\
\text { by means of } \\
\text { collection of } \\
\text { similar attribute } \\
\text { data points }\end{array}$ & $\begin{array}{l}\text { They are } \\
\text { unsupervised } \\
\text { algorithms }\end{array}$ & $\begin{array}{l}\text { Applicable for real } \\
\text { time problems }\end{array}$ & $\begin{array}{l}\text { 1.Identify the correct } \\
\text { inter cluster distance } \\
\text { 2. Identify the total } \\
\text { number of Clusters. } \\
\text { Not suitable for wide } \\
\text { distances. }\end{array}$ \\
\hline $\begin{array}{l}\text { K-means } \\
\text { Clustering }\end{array}$ & $\begin{array}{l}\text { This method is } \\
\text { used when you } \\
\text { have unlabeled } \\
\text { data (i.e. data } \\
\text { without defined } \\
\text { any category) }\end{array}$ & $\begin{array}{l}\text { It clusters, he } \\
\text { given data into } \\
\text { K-clusters or } \\
\text { parts based on } \\
\text { the K-centroids. }\end{array}$ & $\begin{array}{l}\text { Simple to implement. } \\
\text { scalable for large data } \\
\text { sets }\end{array}$ & $\begin{array}{lr}\text { image segments } & \text { are } \\
\text { disconnected } & \text { and } \\
\text { disseminated } & \text { with } \\
\text { wide distance } & \\
\text { dependant on initial } \\
\text { values. }\end{array}$ \\
\hline $\begin{array}{l}\text { Region- } \\
\text { based } \\
\text { methods }\end{array}$ & $\begin{array}{l}\text { Segmentation } \\
\text { based on } \\
\text { similar } \\
\text { properties of } \\
\text { pixels }\end{array}$ & $\begin{array}{l}\text { Partitioning an } \\
\text { image into } \\
\text { homogeneous } \\
\text { regions. }\end{array}$ & $\begin{array}{l}\text { Accurately segment } \\
\text { the regions that have } \\
\text { the same properties we } \\
\text { define. It works well } \\
\text { with respect to noise }\end{array}$ & $\begin{array}{l}\text { Expensive } \\
\text { Computation. } \\
\text { Variation of intensity } \\
\text { will affect the result. }\end{array}$ \\
\hline $\begin{array}{l}\text { Motion \& } \\
\text { Interactive } \\
\text { Segmentat } \\
\text { ion }\end{array}$ & $\begin{array}{l}\text { pixels are } \\
\text { grouping } \\
\text { together in a } \\
\text { particular } \\
\text { movement of } \\
\text { the object }\end{array}$ & $\begin{array}{l}\text { Criminisi } \\
\text { algorithm \& } \\
\text { Watershed } \\
\text { algorithm }\end{array}$ & $\begin{array}{l}\text { The pixels that have } \\
\text { high intensity variation } \\
\text { can be clearly } \\
\text { classified as moving } \\
\text { objects. }\end{array}$ & $\begin{array}{l}\text { If registration of } \\
\text { background pixels are } \\
\text { not perfect, leads false } \\
\text { prediction of moving } \\
\text { objects. More } \\
\text { computation cost. }\end{array}$ \\
\hline $\begin{array}{l}\text { Compress } \\
\text { ion-based } \\
\text { methods }\end{array}$ & $\begin{array}{l}\text { approximation } \\
\text { of actual pixel } \\
\text { values with } \\
\text { some of the } \\
\text { sample pixels }\end{array}$ & $\begin{array}{l}\text { Types: } \\
\text { Lossless, } \\
\text { Compression } \\
\text { Lossy } \\
\text { Compression }\end{array}$ & $\begin{array}{l}\text { Best suited in storage } \\
\text { and transmission of } \\
\text { images }\end{array}$ & $\begin{array}{l}\text { More computation } \\
\text { complexity. }\end{array}$ \\
\hline $\begin{array}{l}\text { Histogram } \\
\text {-based } \\
\text { methods }\end{array}$ & $\begin{array}{l}\text { each region's } \\
\text { pixels are } \\
\text { having similar } \\
\text { properties, like } \\
\text { intensity, color } \\
\text { values, texture. }\end{array}$ & $\begin{array}{l}\text { Best suited for } \\
\text { image } \\
\text { enhancement }\end{array}$ & $\begin{array}{l}\text { Directly process the } \\
\text { color images }\end{array}$ & $\begin{array}{l}\text { Requires high number } \\
\text { of operations on each } \\
\text { pixel. Computationally } \\
\text { slow. Over } \\
\text { segmentation occurred } \\
\text { since no spatial } \\
\text { information. }\end{array}$ \\
\hline
\end{tabular}

\section{Conclusion}

This paper discusses about various image segmentation techniques and different image segmentation approaches are illustrated and correlated. All these methodologies are suitable for many applications in medical field, object detection, computer vision, surveillance system, computer vision etc. From this study, each segmentation methods are desirable for specific image types. So, the combination of multiple segmentation methods is needed to get good result. To improve the accuracy of the segmentation result, need to apply machine learning techniques. The important parameters of image segmentation are accuracy, complexity, efficiency and 
interactivity. No particular methods are comfortable for all the types of images. For this reason, segmentation of images in various applications has high demand in future.

\section{References}

[1] ZhenZhou Wang, "Image segmentation by combining the global and local properties", Elsevier, Expert Systems With Applications (2017), Vol-87, PP- 30-40.

[2] Lahouaoui Lalaoui, Tayeb. Mohamadi and Abdelhak Djaalab, "New Method for Image Segmentation", Elsevier, Procedia - Social and Behavioral Sciences (2015), Vol-195, PP- 1971-1980.

[3] V. Rajinikanth, and M. S. Couceiro, "RGB Histogram based Color Image Segmentation Using Firefly Algorithm", Elsevier, Procedia Computer Science(2015), Vol-46, PP- 1449-1457.

[4] Huang Ying, Li Kai, and Yang Ming, "An Improved Image Inpainting Algorithm based on Image Segmentation", Elsevier, Procedia Computer Science(2017), Vol-107, PP- 796-801.

[5] Gupta Mehul, Patel Ankita, Dave Namrata, Goradia Rahul and Saurin Sheth, "Text-Based Image Segmentation Methodology", Elsevier, Procedia Computer Science (2014), Vol-14, PP- 465-472.

[6] Gupta Mehul, Patel Ankita, Dave Namrata, Goradia Rahul and Saurin Sheth, "Text-Based Image Segmentation Methodology", Elsevier, Procedia Computer Science (2014), Vol-14, PP- 465-472.

[7] Mamta Mittal, Amit Verma, Iqbaldeep Kaur, Bhavneet Kaur, Meenakshi Sharma, Lalit Mohan Goyal, Sudipta Roy and Tai-Hoon Kim, "An Efficient Edge Detection Approach to Provide Better Edge Connectivity for Image Analysis", IEEE Access (2019), Vol-7, PP- 33240-33255.

[8] Ahmed H. Abdel-Gawad, Lobna A. Said, Dave Namrata, and Ahmed G. Radwan, "Optimized Edge Detection Technique for Brain Tumor Detection in MR Images", IEEE Access (2020), Vol-8, PP136243-136259.

[9] Kristina P. Sinaga and Miin-Shen Yang, "Unsupervised K-Means Clustering Algorithm”, IEEE Access (2020), Vol-8, PP- 80716-80727.

[10] Heming Jia Jun Ma and Wenlong Song, "Multilevel Thresholding Segmentation for Color Image Using Modified Moth-Flame Optimization”, IEEE Access (2019), Vol-7, PP- 44097-44134.

[11] Heming Jia Jun Ma and Wenlong Song, "Multilevel Thresholding Segmentation for Color Image Using Modified Moth-Flame Optimization", IEEE Access (2019), Vol-7, PP- 44097-44134.

[12] Ahmed A. Ewees, Mohamed Abd Elaziz, Mohammed A. A. Al-Qaness, Hassan A. Khalil and Sunghwan Kim, “ Two-Step CNN Framework for Text Line Recognition in Camera-Captured Images”, IEEE Access (2020), Vol-8, PP- 26304-26315.

[13] Soosan Beheshti, Edward Nidoy, and Faizan Rahman, "K-MACE and Kernel K-MACE Clustering",IEEE Access (2020), Vol-8, PP- 17390-17403.

[14] Dan Wang, Guoqing Hu, Qianbo Liu, Chengzhi Lyu, and Md Mojahidul Islam, "Region-Based Nonparametric Model for Interactive Image Segmentation”, IEEE Access (2019), Vol-7, PP- 111124111134 .

[15] Jianyu Lin,“A New Perspective on Improving the Lossless Compression Efficiency for Initially Acquired Images", IEEE Access (2019), Vol-7, PP- 144895-144906.

[16] Hongnan Liang, Heming Jia, Zhikai Xing, Jun Ma, And Xiaoxu Peng, "Modified Grasshopper Algorithm-Based Multilevel Thresholding for Color Image Segmentation", IEEE Access (2019), Vol-7, PP- 11258-11295.

[17] Heming Jia, Jun Ma, And Wenlong Song, "Multilevel Thresholding Segmentation for Color Image Using Modified Moth-Flame Optimization", IEEE Access (2019), Vol-7, PP- 44097-44134.

[18] Zhicheng Zhang And Jianqin Yin, "Bee Foraging Algorithm Based Multi-Level Thresholding For Image Segmentation", IEEE Access (2020), Vol-8, PP- 16269-16280.

[19] Zhen Zheng, Bingting Zha, Hailu Yuan, Youshi Xuchen, Yanliang Gao, And He Zhang, "Adaptive Edge Detection Algorithm Based on Improved Grey Prediction Model”, IEEE Access (2020), Vol-8, PP- 102165-102176.

[20] Hanxiao Rong, Alex Ramirez-Serrano, Lianwu Guan, And Yanbin Gao, "Image Object Extraction Based on Semantic Detection and Improved K-Means Algorithm", IEEE Access (2020), Vol-8, PP171129-171139. 